\title{
Ordering of a prototypical conjugated molecular system during monolayer growth on the $(1 \times 2)-A u(110)$ surface
}

\author{
M. Buongiorno Nardelli, ${ }^{*}$ D. Cvetko ${ }^{\dagger}$ V. De Renzi, ${ }^{\ddagger}$ L. Floreano, ${ }^{\S}$ R. Gotter ${ }^{\S}$ A. Morgante, M. Peloi, and F. Tommasini ${ }^{\S}$ \\ Laboratorio TASC dell'Istituto Nazionale di Fisica della Materia, Padriciano 99, 34012 Trieste, Italy \\ R. Danieli, S. Rossini, C. Taliani, and R. Zambonil \\ Istituto di Spettroscopia Molecolare, Consiglio Nazionale delle Richerche, via P. Gobetti 101, 40129 Bologna, Italy
}

(Received 21 September 1995)

\begin{abstract}
The UHV deposition of $\alpha$-hexathiophene (T6) oligomers on the $1 \times 2$ reconstructed $\mathrm{Au}(110)$ surface has been studied in situ. The evolution of the surface morphology during the deposition is followed by the He atom scattering method. A proliferation of Au monoatomic steps is observed during deposition, indicating a strong interaction between Au substrate and T6 molecules. The build-up of a well-ordered single monolayer is obtained with a rectangular unit cell. Its dimensions indicate that $T 6$ molecules lie on the surface, aligned along the rows of close-packed Au atoms.
\end{abstract}

Organic molecules with $\pi$-conjugated electrons have been shown to have unique electronic properties ${ }^{1}$ that offer exciting opportunities for applications in both photonics ${ }^{2}$ and optoelectronics. ${ }^{3}$ In particular, the interaction with and the ordering processes of these molecules at metal surfaces are of basic interest for organic light-emitting diode ${ }^{4}$ technologies. Among organic molecules, $\alpha$-hexathiophene (T6) is a well-characterized prototypical compound for polyconjugated systems. ${ }^{5-12}$ Furthermore, $T 6$ possesses interesting properties of hole injection into highly fluorescent polymers. ${ }^{13}$ It is then of utmost importance, in order to engineer new materials with suitable optoelectronic properties, to understand the essential processes in which $T 6$ molecules form solid structures and to what extent their order can be driven by the substrate structure. In the context of growth mechanisms of organic monolayers, ${ }^{12,14-20}$ it is worth mentioning that organic structures grown quasiepitaxially under ultrahigh vacuum (UHV) have been claimed to show interesting optical properties. ${ }^{21,22}$

In the present work we present a study of the formation of a single molecular layer of $T 6$ by UHV deposition on the $(1 \times 2)-\mathrm{Au}(110)$ surface. He atom scattering has been used to study the surface morphology during deposition. This method has been shown to be particularly suited for studying organic overlayers, ${ }^{23,24}$ being a nonpenetrating and nondestructive technique with a very high surface sensitivity.

The first stages of $T 6$ deposition show substantial modification of the substrate with proliferation of up-down correlated monoatomic steps, but the step density substantially reduces when a highly ordered $T 6$ monolayer with a rectangular unit cell is reached. In this layer, the $T 6$ molecules lie flat on the surface and are aligned along the rows of close packed $\mathrm{Au}$ atoms with a periodicity of $27.4 \pm 0.5 \AA$ and $16.3 \pm 0.2 \AA$, respectively, along the Au rows ( $\Gamma X$ direction) and perpendicular to them ( $\Gamma Y$ direction). The former distance is consistent with the length of a single molecule while the latter is twice that of the $(1 \times 2)$-Au(110) missing row structure.

The He beam apparatus, described in detail elsewhere, ${ }^{25}$ has a fixed scattering geometry with angle of incidence $\theta_{i}=55^{\circ}-\alpha$ and exit angle $\theta_{\text {out }}=55^{\circ}+\alpha$. The sample is mounted on a high-precision manipulator with six degrees of freedom and with an angular reproducibility better than $0.01^{\circ}$. The diffraction intensities are collected by scanning the angle $\alpha$, which varies the parallel momentum exchange as $\Delta K_{\|}=2 k \cos \left(55^{\circ}\right) \sin (\alpha)$, where $k$ is the modulus of the He wave vector. The He beam energy can be selected between 19 and $65 \mathrm{meV}\left(k=6.06\right.$ and $11.3 \AA^{-1}$, respectively) and the energy spread is $0.3 \mathrm{meV}$ at the lowest beam energy. The $\mathrm{Au}(110)$ sample was prepared by usual sputteringannealing procedure ${ }^{26}$ and it demonstrated a sharp $(1 \times 2)$ diffraction pattern characteristic of the $\mathrm{Au}$ missing-row reconstruction. $^{27,28}$ Its unit-cell vectors are $x=2.88 \AA$ and $y=8.16 \AA$, respectively, along $\Gamma X$ and $\Gamma Y$. The average size of the flat $(1 \times 2)$ domains was determined to be 500 and $300 \AA$, respectively, along the rows of close-packed Au atoms and perpendicular to the rows. ${ }^{26} T 6$ was synthesized as reported in Ref. 6 and the purified polycrystalline powder was placed in a crucible in the UHV chamber.

The $T 6$ beam, sublimated from the crucible, reached the surface at a grazing angle of $\sim 20^{\circ}$ with a growth rate of $\simeq 0.005 \mathrm{ML} / \mathrm{min}$. The surface temperature during deposition was selected at $375 \mathrm{~K}^{29}$ At this temperature the mobility of T6 molecules is high enough to form correlated structures over the surface and this morphology is reached almost instantaneously for a given coverage. No further evolution of surface structures can be observed after interruption of $T 6$ deposition, which confirms that the deposition process at this temperature proceeds through a series of quasiequilibrium surface structures. Moreover, the diffraction intensities of both the bare Au surface and the $T 6$-covered surface remain stable for several days at a base pressure of $5 \times 10^{-11} \mathrm{mbar}$, indicating that surface contamination is completely negligible. The surface evolution during deposition was followed by taking angular profiles along the $\Gamma Y$ direction with the $\mathrm{He}$ beam energy fixed at $19 \mathrm{meV}$, as shown in Fig. 1. The halfinteger Bragg peak observed for the bare surface (curve $a$ ) is due to the $(1 \times 2)$ missing-row reconstruction and disappears in the early stages of deposition when the $T 6$ coverage is as 


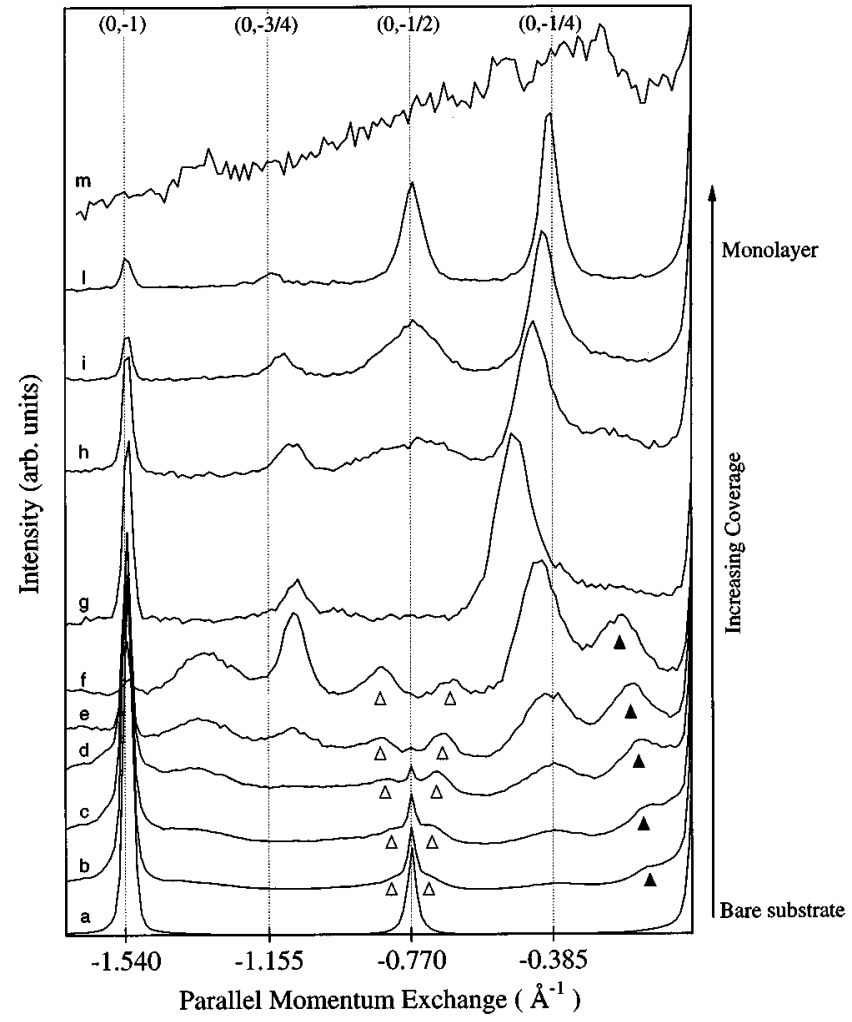

FIG. 1. Angular profiles taken along $\Gamma Y$ during deposition at $T=375^{\circ} \mathrm{K}$ with a He beam energy of $19.0 \mathrm{meV}$. All curves are normalized to the specular intensity and are shifted upward for clarity, the arrow on the right side of the figure indicates the direction of increasing $T 6$ coverage. Curve $a$ corresponds to the bare $(1 \times 2)-\mathrm{Au}(110)$ surface. Filled and open triangles indicate the position of the side peaks associated to the specular and the half-order peaks, respectively.

low as $0.1 \mathrm{ML}{ }^{30}$ Subsequent scans shown in Fig. 1 present very rich $\Gamma Y$ patterns, revealing that $T 6$ deposition induces various correlated surface structures and that eventually a well-ordered phase is reached displaying diffraction peaks at quarter integer order position (see curve $l$ in Fig. 1).

For this structure, the diffraction pattern taken along $\Gamma X$ presents weak but well-resolved diffraction peaks corresponding to a lattice vector equal to $27.4 \pm 0.5 \AA$, closely matching the length of the $T 6$ molecule. This leads us to the conclusion that the final ordered $T 6$ structure consists of $T 6$ molecules lined up along $\Gamma X$. They are in register with the substrate along $\Gamma Y$ (see curve $l$ in Fig. 1), and we can label this structure as $(T \times 4) T 6-\mathrm{Au}(110)$ with $T=(27.4 / x)$. It is further noted that the average ordered $(T \times 4)$ domain, as evaluated by the diffraction peak width, extends at least 150 and $100 \AA$, respectively along the $\Gamma Y$ and $\Gamma X$ directions. Further $T 6$ deposition after formation of the $(T \times 4)$ layer (see curve $m$ in Fig. 1), results in a continuous decrease of all diffracted peaks up to their disappearance. This indicates that no other ordered structures are formed after the $(T \times 4)$ one, which then corresponds to the completion of a single monolayer. Two possible models can be adopted to explain the fourfold periodicity along $\Gamma Y$, which is doubled with respect to the Au missing-row structure. The $(T \times 4)$ unit cell could be built up either by only one $T 6$ molecule within the surface a)

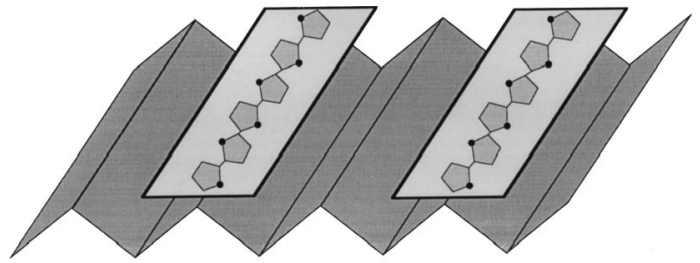

b)

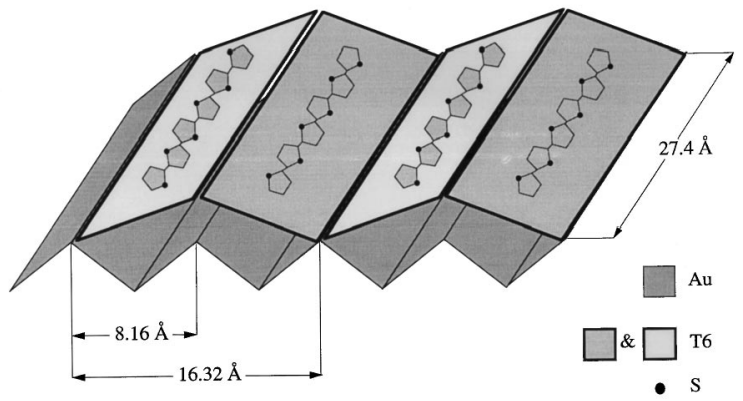

FIG. 2. Sketches of the two possible models describing the $T \times 4$ structure. The dark gray regions represent the $(1 \times 2)-\mathrm{Au}(110)$ surface, while the light gray rectangles depict $T 6$ molecules. The dimensions of the $T \times 4$ unit cell are indicated. The distance $8.16 \AA$ refers to the bare $(1 \times 2)-A u(110)$ surface. In sketch (b) the different shadowing of molecules visualizes their inequivalence with respect to He diffraction.

unit cell, or by two of them displaying different tilting angle with respect to the surface normal as depicted in Figs. 2(a) and 2(b). The choice between these two microscopic models for the $(T \times 4)$ structure is only speculative but it should be noted that the ratio between the $\Gamma Y$ diffraction intensities and the specular intensity is much lower for the $(T \times 4)$ structure than for the bare substrate, suggesting that the $T 6$ molecules have filled the grooves of the missing-rows $\mathrm{Au}$ structure. $^{31}$

The diffraction patterns along $\Gamma Y$ at an intermediate state of deposition (see curves $b-f$ in Fig. 1) are characterized by the appearence of features aside from the specular and the half-order Au Bragg peaks, hereafter referred to as side peaks and indicated in Fig. 1 with filled and open triangles, respectively. As we shall see, these side peaks are related to the proliferation of $\mathrm{Au}$ monoatomic steps on the surface and their angular profiles reflect the distribution of equivalent terraces. ${ }^{32}$ Two terraces are meant to be equivalent if they scatter the He waves with a phase difference $\Phi=2 n \pi$. $\Phi$ depends both on the momentum exchange and on the total displacement introduced by steps between terraces. It is given by $\Phi=\Delta K_{\|} \Sigma_{i} d_{i}+\Delta K_{\perp} \Sigma_{i} t_{i}$, where $t_{i}$ and $d_{i}$ are, respectively, the vertical and the lateral component of the $i$ th step displacement on the surface. For Au steps $t=1.44 \AA$, while $d=(3 / 4) a_{1 \times 2}$ in the $\Gamma Y$ direction. ${ }^{33}$ For the specular peak when $\Delta K_{\perp}=(2 n+1) \pi / t \quad$ (antiphase condition), equivalent terraces are those separated by two subsequent steps. Instead, for the half-order peak, equivalent terraces are those separated by four steps, as each step introduces a phase shift $\Phi_{i}=(3 / 2) \pi$. As shown in Fig. 3, we have taken several angular profiles along $\Gamma Y$ with different beam energies. The specular side-peak intensity as compared to the Bragg-peak 


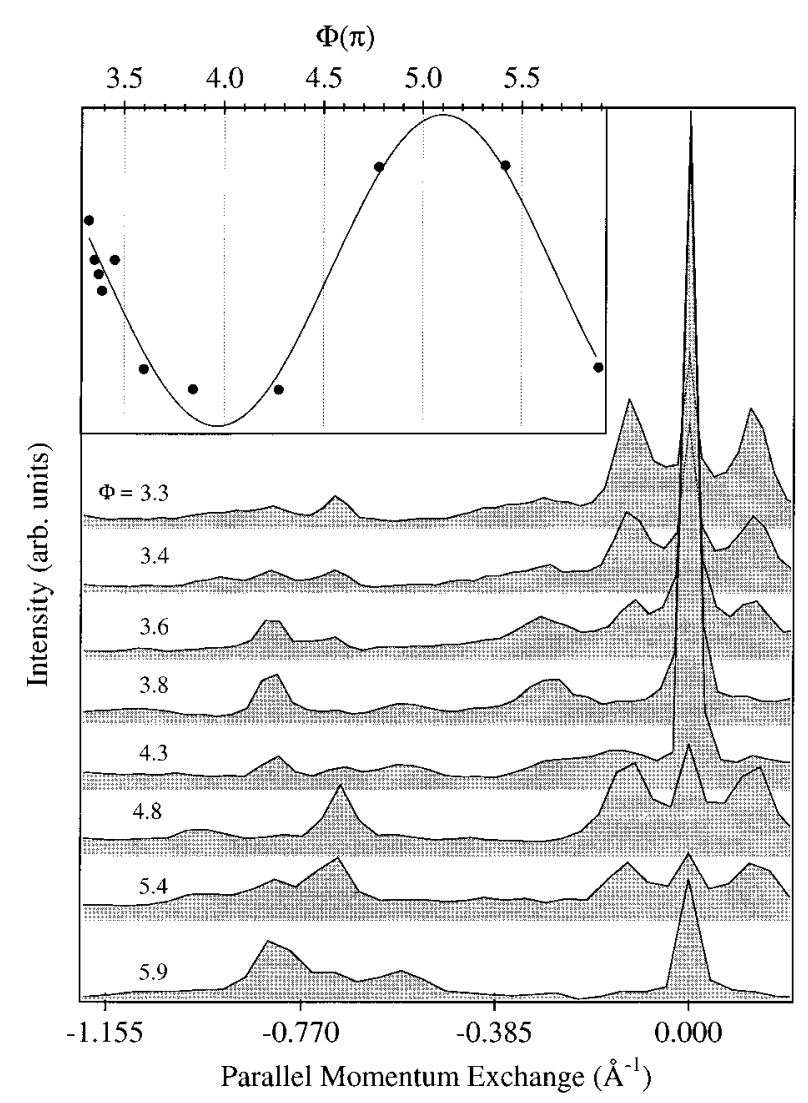

FIG. 3. Angular profiles taken along $\Gamma Y$ for different He beam energies varying from 19 to $65 \mathrm{meV}$. $T 6$ coverage can be estimates to $0.2 \pm 0.05 \mathrm{ML}$. $\Phi$ denotes the phase condition $\Delta k_{\perp} \cdot t$ for the specular peak. The inset shows the ratio between the side peaks and the specular intensities as a function of $\Phi$. The full line is fitted to the sine function and shows how minima correspond to even $\Phi$ values (in phase), while maxima correspond to odd ones (antiphase).

intensity is seen to oscillate with $\Delta K_{\perp}$. From the $\Delta K_{\perp}$ values for antiphase (maximum) and in-phase (minimum) conditions we evaluated a step vertical displacement of $1.4 \AA$, in good agreement with the $\mathrm{Au}(110)$ value reported in the literature. This oscillating behavior also indicates that terraces are distributed over only two adjacent levels, namely, steps are up-down correlated. ${ }^{34}$ Moreover, it is important to notice that the shape of the specular angular profile indicates that the distribution of distance between equivalent terraces is peaked at a most probable value $\Lambda_{0}=2 \pi / Q_{\max }$, where $\pm Q_{\max }$ are the positions of the specular side-peak maxima (filled triangles in Fig. 1). Instead, the distance of the halforder side peaks (open triangles in Fig. 1) from the Bragg position results to be $Q_{1 \times 2}=1 / 2 Q_{\max }$. This means that the most probable distance between equivalent terraces for the half-order diffraction peak is twice that for the specular one, proving definitively the presence of steps induced by $T 6$ in the early stages of deposition. $T 6$ therefore induces the previously flat Au surface to deconstruct. We speculate that the charge redistribution between substrate and oligomers encourages step formation. This is consistent with the observed step density increase with $T 6$ coverage (see the shift of the side-peak positions in Fig. 1).

Diffraction features other than appearing in the first stages of growth (curves $b-f$ in Fig. 1) should be related to some incommensurate ordering of oligomers as deposition increases. Their position can vary slightly from run to run, probably due to small difference in the $T 6$ deposition rate from run to run. Nevertheless, for a coverage of $\simeq 0.7 \mathrm{ML}$ (curve $g$ in Fig. 1) the diffraction pattern along $\Gamma Y$ always displays a quasicommensurate threefold structure characterized by the $(0, \pm 1 / 3)$ and the $(0, \pm 2 / 3)$ peaks, which deviate from their commensurate position towards the nearest integer order peaks. This threefold structure eventually tranforms into a fourfold structure where the $(0,2 / 4)$ peak appears and the third-order Bragg peaks shift toward fourth-order positions (Fig. 1, curves $h-l$ ).

Finally, we comment briefly on the ordering process along the $\Gamma X$ direction. No diffraction peaks or features were observed along $\Gamma X$ until the final $(T \times 4)$ structure was achieved, showing the one-dimensional character of this ordering process. This fact points out the prominent role of the $(1 \times 2)$ surface anisotropy in driving the overlayer growth.

In conclusion, we have shown the formation and ordering of a single monolayer of the prototypical model compound $T 6$ on a $(1 \times 2)$ reconstructed $\mathrm{Au}(110)$ surface. T6 molecules line up with the long molecular axis parallel to the surface along the $\Gamma X$ substrate direction, displaying an ordered $(T \times 4)$ structure. It is worth noting how different is the first $T 6$ monolayer structure from the bulk threedimensional $T 6$ crystalline form, which has been found by $\mathrm{x}$-ray scattering to order in a monoclin unit cell belonging to the $P_{2_{l}} / n$ space group and packing with a herringbone structure. ${ }^{35,36}$ Moreover, oligothiophene molecules deposited in thin films on polycrystalline or amorphous substrates are reported to grow in a completely different way, ${ }^{12,37,38}$ resulting from a self-assembly ordering process of oligothiophenes.

We have also shown that there is a mutual influence between substrate and oligomers. That is, on the one hand, for early stages of deposition $T 6$ induces a proliferation of updown correlated Au monoatomic steps. On the other hand, the substrate structure - in particular its anisotropy drives the ordering of the overlayer, characterized by a preferred orientation of the molecules parallel to the substrate troughs. As the electronic and optical properties of oligomeric films strongly depends on their structure, the $(T \times 4) T 6-\mathrm{Au}(110)$ system may possibly display peculiar characteristics. Moreover, the strong interaction between $T 6$ and gold surface indicates a different electronic rearrangement of the gold-T6 interface. Work function, mobility, charge injection, and linear and nonlinear optical properties will be consequently affected. The understanding and the possible tailoring of the interface properties are of fundamental importance for photonics and optoelectronics applications, where these rigid rodlike molecules have been demonstrated to be promising new active materials.

This work was partly supported by the EEC ESPRIT Basic Research Action 8013-LEDFOS. 
*Also at Bull-Unix Competence Center, AREA di Ricerca di Trieste, Padriciano 99, I-34012 Trieste, Italy.

†Permanent address: J. Stefan Institute, University of Ljubljana, Slovenia.

${ }^{\ddagger}$ Also at Dipartimento di Fisica dell’Università di Modena, Italy.

${ }^{\S}$ Also at Dipartimento di Fisica dell’Università di Trieste, Italy.

"Author to whom correspondence should be addressed.

${ }^{1}$ Introduction to Molecular Electronics, edited by M. C. Petty, M. R. Bryce, and D. Bloor (Edward Arnolds, London, 1995).

${ }^{2}$ Nonlinear Optical Effects in Organic Polymers, Vol. 162 of NATO Advanced Study Institute, Series E, edited by J. Messier, F. Kajzar, P. Prasad, and D. Ulrich (Kluwer Academic, Dordrecht, 1990).

${ }^{3}$ Conjugated Polymeric Materials: Opportunities in Electronics, Optoelectronics and Molecular Electronics, Vol. 182 of NATO Advanced Study Institute, Series E, edited by J. L. Breadas and R. R. Chance (Kluwer Academic, Dordrecht, 1990).

${ }^{4}$ J. H. Burroughes, D. D. C. Bradley, A. R. Brown, R. N. Marks, K. Mackay, R. H. Friends, P. L. Burns, and A. B. Holmes, Nature 347, 539 (1990).

${ }^{5}$ N. Periasamy, R. Danieli, G. Ruani, R. Zamboni, and C. Taliani, Phys. Rev. Lett 68, 919 (1992).

${ }^{6}$ R. Zamboni, N. Periasamy, G. Ruani, and C. Taliani, Synth. Met. 54, 57 (1993).

${ }^{7}$ D. Fichou, G. Horowitz, B. Hu, and F. Garnier, Synth. Met. 48, 167 (1992).

${ }^{8}$ L. M. Blinov, S. P. Palto, G. Ruani, C. Taliani, A. A. Tevosov, S. G. Yudin, and R. Zamboni, Chem. Phys. Lett. 232, 401 (1995).

${ }^{9}$ G. Horowitz, X. Z. Peng, D. Fichou, and F. Garnier, J. Appl. Phys. 67, 528 (1990).

${ }^{10}$ H.-J. Egelhaaf, P. Bäuerle, K. Rauer, V. Hoffmann, and D. Oelkrug, Synth. Met. 61, 143 (1993).

${ }^{11}$ P. Dannetun, M. Borman, S. Stafstrom, W. R. Salaneck, R. Lazzaroni, C. Friedriksson, J. L. Bredas, R. Zamboni, and C. Taliani, J. Chem. Phys. 99, 664 (1993).

${ }^{12}$ K. Hamano, T. Kurata, S. Kubota, and H. Koezuka, Jpn. J. Appl. Phys. 33, 1031 (1994).

${ }^{13}$ F. Cacialli, R. N. Marks, R. H. Friends, R. Zamboni, C. Taliani, S. C. Moratti, and A. B. Holmes (unpublished).

${ }^{14}$ H. Tada, K. Saiki, and A. Koma, Jpn. J. Appl. Phys. 30, L306 (1991)

${ }^{15}$ C. Ludwig, B. Gomph, W. Glatz, J. Petersen, W. Eisenmenger, M. Mobus, U. Zimmerman, and N. Karl, Z. Phys. B 86, 397 (1992).

${ }^{16}$ P. Fenter, P. E. Burrows, P. Eisenberger, and S. R. Forrest, J. Cryst. Growth 152, 65 (1995).
${ }^{17}$ P. E. Burrows, Y. Zhang, E. I. Haskal, and S. R. Forrest, Appl. Phys. Lett. 61, 2417 (1992).

${ }^{18}$ K. Ishida, K. Hayashi, T. Horiuchi, and K. Matsushige, J. Appl. Phys. 73, 7338 (1993).

${ }^{19}$ K. Ishida, T. Horiuchi, S. Kay, and K. Matsushige, J. Appl. Phys. 34, L240 (1995).

${ }^{20} \mathrm{~S}$. Taki, K. Ishida, H. Okabe, and K Matsushige, J. Cryst. Growth 131, 13 (1993).

${ }^{21}$ F. F. So, S. R. Forrest, Y. Q. Shi, and W. H. Steier, Appl. Phys. Lett. 56, 674 (1990).

${ }^{22}$ V. M. Agranovich, R. D. Atanasov, and G. F. Bassani, Chem. Phys. Lett. 199, 6 (1992).

${ }^{23}$ N. Camillone III, C. E. D. Chidsey, G.-Y. Liu, and G. Scoles, J. Chem. Phys. 98, 3503 (1993).

${ }^{24}$ G. Witte, H. Range, J. P. Toennies, and Ch. Woll, Phys. Rev. Lett. 71, 1063 (1993).

${ }^{25}$ D. Cvetko, A. Lausi, A. Morgante, F. Tommasini, K. C. Prince, and M. Sastry, Meas. Sci. Technol. 3, 997 (1992).

${ }^{26}$ D. Cvetko, A. Lausi, A. Morgante, F. Tommasini, and K. C. Prince, Surf. Sci. 269/70, 68 (1992).

${ }^{27}$ G. Binning, H. Rohrer, Ch. Gerber, and E. Weibel, Surf. Sci. 131, L379 (1983).

${ }^{28}$ W. Moritz and D. Wolf, Surf. Sci. 163, L655 (1986).

${ }^{29}$ For this temperature the long-range order of the monolayer structure is substantially better than for room temperature, while for higher temperatures the Debye-Waller damping of diffraction peaks intensities hinders the measurements during growth.

${ }^{30}$ The absolute coverage of the $T 6$ overlayer is assumed to be proportional to deposition time.

${ }^{31}$ In first approximation, the modulation of the diffracted peak intensities reflects the surface corrugation. The $(T \times 4)$ structure results to be less corrugated than the bare- $(1 \times 2)-\mathrm{Au}(110)$ one.

${ }^{32}$ J. Lapujoulade, in Helium Atom Scattering from Surfaces, edited by E. Hulpke, Springer Series in Surface Sciences Vol. 27 (Springer, Berlin, 1992), p. 95, and references therein.

${ }^{33}$ P. Fenter, and T.-M. Lu, Surf. Sci. 154, 15 (1985).

${ }^{34}$ C. S. Lent and P. I. Cohen, Surf. Sci. 139, 121 (1984), and references therein.

${ }^{35}$ W. Porzio, S. Destri, M. Mascherpa, and S. Bruckner, Acta Polym. 44, 266 (1993).

${ }^{36}$ G. Horowitz, B. Bachet, A. Yassar, P. Lang, F. DeManze, J. L. Fave, and F. Garnier, Chem. Mater. 7, 1337 (1995).

${ }^{37}$ H.-J. Egelhaaf, P. Bäuerle, K. Rauer, V. Hoffmann, and D. Oelkrug, J. Mol. Struct. 293, 249 (1993).

${ }^{38}$ O. Böhme, C. Ziegler, and W. Göpel, Adv. Mater. 6, 587 (1994). 